\title{
The wave equation with locally distributed control in non-cylindrical domain
}

\section{Lizhi Cui $^{{ }^{*}}$}

"Correspondence:

cuilz924@126.com

'College of Applied Mathematics,

Jilin University of Finance and

Economics, Changchun, China

\begin{abstract}
This paper is concerned with exact internal controllability for a one-dimensional wave equation in a non-cylindrical domain. This equation characterizes the motion of a string with a fixed endpoint and the other moving one. When the speed of the moving endpoint is less than wave speed, exact internal controllability of this equation is established.
\end{abstract}

Keywords: Locally distributed control; Wave equation; Non-cylindrical domain

\section{Introduction}

Given $T>0$. For any $0<k<1$, set

$$
\alpha_{k}(t)=1+k t \quad \text { for } t \in[0, T] .
$$

Also, define the following non-cylindrical domains:

$$
\widehat{Q}_{T}^{k}=\left\{(x, t) \in \mathbb{R}^{2} ; 0<x<\alpha_{k}(t), t \in[0, T]\right\}
$$

and for any $0<m^{\prime}<m<\frac{n}{2}<n<n^{\prime}<1$,

$$
\begin{aligned}
& \widehat{Q_{1}}=\left\{(x, t) \in \mathbb{R}^{2} ; m \alpha_{k}(t)<x<n \alpha_{k}(t), t \in[0, T]\right\}, \\
& \widehat{Q_{2}}=\left\{(x, t) \in \mathbb{R}^{2} ; m^{\prime} \alpha_{k}(t)<x<n^{\prime} \alpha_{k}(t), t \in[0, T]\right\} .
\end{aligned}
$$

Let

$$
H^{1}\left(\widehat{Q_{1}}\right)=\left\{f \in L^{2}\left(\widehat{Q_{1}}\right) \mid \frac{\partial f}{\partial x}, \frac{\partial f}{\partial t} \in L^{2}\left(\widehat{Q_{1}}\right)\right\},
$$

and we denote by $\left[H^{1}\left(\widehat{Q_{1}}\right)\right]^{\prime}$ the dual space to $H^{1}\left(\widehat{Q_{1}}\right)$, i.e., $\left[H^{1}\left(\widehat{Q_{1}}\right)\right]^{\prime}=\left\{g: H^{1}\left(\widehat{Q_{1}}\right) \rightarrow\right.$ $R ; g$ is bounded linear $\}$.

(c) The Author(s) 2019. This article is distributed under the terms of the Creative Commons Attribution 4.0 International License (http://creativecommons.org/licenses/by/4.0/), which permits unrestricted use, distribution, and reproduction in any medium, provided you give appropriate credit to the original author(s) and the source, provide a link to the Creative Commons license, and indicate if changes were made. 
In this paper, we consider the following control problem associated with the onedimensional wave equation:

$$
\begin{cases}u_{t t}-u_{x x}=B v & \text { in } \widehat{Q}_{T}^{k}, \\ u(0, t)=u\left(\alpha_{k}(t), t\right)=0 & \text { on }(0, T), \\ u(x, 0)=u^{0}, \quad u_{t}(x, 0)=u^{1} & \text { in }(0,1),\end{cases}
$$

where $u$ is the state variable, $\left(u^{0}, u^{1}\right) \in L^{2}(0,1) \times H^{-1}(0,1)$ is any given initial value, $v \in$ $\left[H^{1}\left(\widehat{Q_{1}}\right)\right]^{\prime}$ is the control variable, the constant $k$ is called speed of the moving endpoint and $B \in C^{\infty}\left(\widehat{Q}_{T}^{k}\right)$,

$$
B(x, t) \begin{cases}=0, & (x, t) \in \widehat{Q}_{T}^{k} \backslash \widehat{Q_{2}}, \\ =1, & (x, t) \in \widehat{Q_{1}}, \\ \in(0,1), & (x, t) \in \widehat{Q_{2}} \backslash \widehat{Q_{1}} .\end{cases}
$$

By [1], it is easy to check that (1.2) has a unique weak solution

$$
u \in C\left([0, T] ; L^{2}\left(0, \alpha_{k}(t)\right)\right) \cap C^{1}\left([0, T] ; H^{-1}\left(0, \alpha_{k}(t)\right)\right) .
$$

The problem of exact controllability for (1.2) is formulated as follows.

Definition 1.1 (1.2) is called exactly controllable at the time $T$ if for any initial value $\left(u^{0}, u^{1}\right) \in L^{2}(0,1) \times H^{-1}(0,1)$ and any target $\left(u_{d}^{0}, u_{d}^{1}\right) \in L^{2}\left(0, \alpha_{k}(T)\right) \times H^{-1}\left(0, \alpha_{k}(T)\right)$, one can always find a control $v \in\left[H^{1}\left(\widehat{Q_{1}}\right)\right]^{\prime}$ such that the corresponding weak solution $u$ of (1.2) satisfies

$$
u(T)=u_{d}^{0}, \quad u_{t}(T)=u_{d}^{1} .
$$

The main goal of this article is to obtain the exact controllability of (1.2). In practical situations, many processes evolve in domains whose boundaries have moving parts. A simple model, e.g., is interface of ice water mixture when the temperature rises. To study controllability problem of wave equations with moving boundary or free boundary is very meaningful. As we all know, there exist numerous literature works on the controllability problems of wave equations in a cylindrical domain, see e.g. [2-7]. However, there are only a few works on the exact controllability for wave equations defined in non-cylindrical domains. We refer to [8-16] for some known results in this respect. In [8-13], boundary controllability for wave equations with moving boundary was obtained. In [15], distributed controllability of a wave equation with constant coefficients in a non-cylindrical domain was established, when a control entered the system through the whole non-cylindrical domain. While in [16], locally distributed control of a one-dimensional wave equation in a non-cylindrical domain was obtained when $k \in(0, \tilde{k}), 0<\tilde{k}<1$. Motivated by [13-16], we extend the result in [16], and locally distributed control is obtained when $k \in(0,1)$. The key point is to define directly the energy function of a wave equation in the non-cylindrical domain and use the multiplier method to overcome these difficulties.

Our paper is divided into three sections. In Sect. 2, we state the principal result. In Sect. 3, using the multiplier method, we consider the homogeneous wave equation and establish observability inequality. 


\section{Preliminaries and main results}

Lemma 2.1 Let $X$ be a Banach space, and let $A: X \rightarrow Y$ be a bounded linear operator. Then the following properties are equivalent: (a) $R(A)=Y$, (b) $A^{\prime}$ has a continuous inverse operator.

Set $T_{k}^{*}>0$ for the controllability time. The main result of this paper is stated as follows.

Theorem 2.1 Let $0<k<1$ and $T>T_{k}^{*},(1.2)$ is exactly controllable at time $T$ in the sense of Definition 1.1.

Remark 2.1 $T_{k}^{*}$ will be defined during the course of the later proof.

Remark 2.2 We can obtain the same result as that in this paper for a more general function $\alpha_{k}(t)$ as long as it meets the condition $0<\alpha_{k}^{\prime}(t)<1$.

Remark 2.3 We expect to obtain the same result in the forthcoming paper, when the control variable $v \in L^{2}(0, T ; \omega(t)), \omega(t) \subseteq\left(0, \alpha_{k}(t)\right)$.

To prove this, let $u=\xi+\eta$, where $\xi$ and $\eta$ satisfy the following systems:

$$
\begin{aligned}
& \begin{cases}\xi_{t t}-\xi_{x x}=0, & (x, t) \in \widehat{Q}_{T}^{k}, \\
\xi(0, t)=\xi\left(\alpha_{k}(t), t\right)=0, & t \in(0, T), \\
\xi(x, 0)=u^{0}, \quad \xi_{t}(x, 0)=u^{1}, & x \in(0,1),\end{cases} \\
& \begin{cases}\eta_{t t}-\eta_{x x}=B v, & (x, t) \in \widehat{Q}_{T}^{k}, \\
\eta(0, t)=\eta\left(\alpha_{k}(t), t\right)=0, & t \in(0, T), \\
\eta(x, 0)=\eta_{t}(x, 0)=0, & x \in(0,1) .\end{cases}
\end{aligned}
$$

Therefore, we only need to obtain internal controllability of (2.2).

Theorem 2.2 Let $T>T_{k}^{*}$. Then, for any target $\left(u_{d}^{0}, u_{d}^{1}\right) \in L^{2}\left(0, \alpha_{k}(T)\right) \times H^{-1}\left(0, \alpha_{k}(T)\right)$, there exists a control $v \in\left[H^{1}\left(\widehat{Q_{1}}\right)\right]^{\prime}$ such that the corresponding weak solution $\eta$ of $(2.2)$ satisfies

$$
\eta(T)=u_{d}^{0}, \quad \eta_{t}(T)=u_{d}^{1}
$$

Remark 2.4 If Theorem 2.2 holds, then Theorem 2.1 holds. In fact, for any $\left(u^{0}, u^{1}\right) \in$ $L^{2}(0,1) \times H^{-1}(0,1)$ and any target $\left(u_{d}^{0}, u_{d}^{1}\right) \in L^{2}\left(0, \alpha_{k}(T)\right) \times H^{-1}\left(0, \alpha_{k}(T)\right),\left(u_{d}^{0}-\xi(T), u_{d}^{1}-\right.$ $\left.\xi_{t}(T)\right)$ belongs to $L^{2}\left(0, \alpha_{k}(T)\right) \times H^{-1}\left(0, \alpha_{k}(T)\right)$, where $\xi$ is the solution of (2.1) associated to $\left(u^{0}, u^{1}\right)$. By Theorem 2.2, we choose $v \in\left[H^{1}\left(\widehat{Q_{1}}\right)\right]^{\prime}$, then $\eta$ satisfies

$$
\eta(T)=u_{d}^{0}-\xi(T), \quad \eta_{t}(T)=u_{d}^{1}-\xi_{t}(T)
$$

This implies that $u=\xi+\eta$ satisfies (1.2) and (1.4).

In the following, we prove Theorem 2.2. Let us introduce some notations. Write $U=$ $\left[H^{1}\left(\widehat{Q_{1}}\right)\right]^{\prime}, F=L^{2}\left(0, \alpha_{k}(T)\right) \times H^{-1}\left(0, \alpha_{k}(T)\right)$ and $F^{\prime}=H_{0}^{1}\left(0, \alpha_{k}(T)\right) \times L^{2}\left(0, \alpha_{k}(T)\right)$. Then we 
define the scalar products between $F$ and $F^{\prime}$ :

$$
\begin{aligned}
& \left\langle\left(w(x, T), w_{t}(x, T)\right),\left(z(x, T), z_{t}(x, T)\right)\right\rangle_{F, F^{\prime}} \\
& \quad=\int_{0}^{\alpha_{k}(T)} w_{t}(x, T) z(x, T) d x-\int_{0}^{\alpha_{k}(T)} w(x, T) z_{t}(x, T) d x,
\end{aligned}
$$

where for any $\left(w(x, T), w_{t}(x, T)\right) \in F$ and any $\left(z(x, T), z_{t}(x, T)\right) \in F^{\prime}$.

Define a linear operator $A$ :

$$
\begin{aligned}
& A: U \rightarrow F, \\
& A v=\left(\eta(x, T), \eta_{t}(x, T)\right) \quad \forall v \in U,
\end{aligned}
$$

where we use $\eta$ to denote the solution of (2.2) associated to $v$. Then $A$ is surjective equivalent to internal controllability of the wave equation (2.2). By Lemma 2.1 , we only prove that $A^{\prime}$ has a continuous inverse operator. Now we define $A^{\prime}$. $A^{\prime}$ is associated with the following homogeneous wave equation:

$$
\begin{cases}z_{t t}-z_{x x}=0, & (x, t) \in \widehat{Q}_{T}^{k}, \\ z(0, t)=z\left(\alpha_{k}(t), t\right)=0, & t \in(0, T), \\ z(x, T)=z^{0}, \quad z_{t}(x, T)=z^{1}, & x \in\left(0, \alpha_{k}(T)\right),\end{cases}
$$

where $\left(z^{0}, z^{1}\right) \in H_{0}^{1}\left(0, \alpha_{k}(T)\right) \times L^{2}\left(0, \alpha_{k}(T)\right)$ is any given initial value. (2.3) has a unique weak solution

$$
z \in C\left([0, T] ; H_{0}^{1}\left(0, \alpha_{k}(t)\right)\right) \cap C^{1}\left([0, T] ; L^{2}\left(0, \alpha_{k}(t)\right)\right) .
$$

Multiplying the first equation of (2.2) by $z$ and integrating on $\widehat{Q}_{T}^{k}$, we have

$$
\begin{aligned}
\langle B v, z\rangle & =\int_{0}^{\alpha_{k}(T)} \eta_{t}(x, T) z^{0} d x-\int_{0}^{\alpha_{k}(T)} \eta(x, T) z^{1} d x \\
& =\left\langle\left(\eta(x, T), \eta_{t}(x, T)\right),\left(z^{0}, z^{1}\right)\right\rangle \\
& =\left\langle A v,\left(z^{0}, z^{1}\right)\right\rangle .
\end{aligned}
$$

Let $B^{\prime}$ be the adjoint operator of $B$ in (1.3) and if $v \in\left[H^{1}\left(\widehat{Q_{1}}\right)\right]^{\prime}$, then

$$
B^{\prime}: H^{1}\left(\widehat{Q}_{T}^{k}\right) \rightarrow H^{1}\left(\widehat{Q_{1}}\right)
$$

from which we have

$$
\left\langle v, B^{\prime} z\right\rangle=\left\langle v, A^{\prime}\left(z^{0}, z^{1}\right)\right\rangle
$$

Hence $A^{\prime}$ is defined as follows:

$$
A^{\prime}\left(z^{0}, z^{1}\right)=B^{\prime} z(x, t)=z(x, t), \quad(x, t) \in \widehat{Q_{1}}, \forall\left(z^{0}, z^{1}\right) \in F^{\prime},
$$


where $z$ is the solution of (2.3). Therefore internal controllability of the wave equation (2.2) is equivalent to the following inequality:

$$
|z|_{H^{1}\left(\widehat{Q_{1}}\right)} \geq C\left|\left(z^{0}, z^{1}\right)\right|_{F^{\prime}} \quad \forall\left(z^{0}, z^{1}\right) \in F^{\prime}
$$

In the sequel, we denote by $C$ a positive constant depending only on $T$ and $k$, which may be different from one place to another.

\section{Observability inequality of wave equations}

In the following, we shall give proof of (2.4) by the multiplier method. The energy function of system of (2.3) is defined as follows:

$$
E(t)=\frac{1}{2} \int_{0}^{\alpha_{k}(t)}\left[\left|z_{t}(x, t)\right|^{2}+\left|z_{x}(x, t)\right|^{2}\right] d x \quad \text { for } t \geq 0
$$

where $z$ is the solution of (2.3). In particular,

$$
E_{T}=\frac{1}{2} \int_{0}^{\alpha_{k}(T)}\left[\left|z^{1}(x)\right|^{2}+\left|z_{x}^{0}(x)\right|^{2}\right] d x
$$

By similar method, we obtain the following lemma about a growth estimate of the energy function (see the detailed proof in [13]).

Lemma 3.1 For any $\left(z^{0}, z^{1}\right) \in H_{0}^{1}\left(0, \alpha_{k}(T)\right) \times L^{2}\left(0, \alpha_{k}(T)\right)$ and $t \in[0, T]$, the corresponding solution $z$ of (2.3) follows:

$$
\frac{(1-k) \alpha_{k}(T)}{(1+k) \alpha_{k}(t)} E_{T} \leq E(t) \leq \frac{(1+k) \alpha_{k}(T)}{(1-k) \alpha_{k}(t)} E_{T} .
$$

Remark 3.1 From Lemma 3.1, we obtain that

$$
\frac{(1-k)}{(1+k) \alpha_{k}(t)} E_{T} \leq E(t) \leq \frac{(1+k)}{(1-k)} \alpha_{k}(T) E_{T}
$$

and

$$
\frac{(1-k) \alpha_{k}(T)}{(1+k)} E_{T} \leq E(0) \leq \frac{(1+k) \alpha_{k}(T)}{(1-k)} E_{T} .
$$

For any $0 \leq t \leq T$, let $p \in \operatorname{Lip}\left[0, \alpha_{k}(t)\right]$ and

$$
p(x, t)= \begin{cases}-(n-m) x, & x \in\left[0, m \alpha_{k}(t)\right], \\ {[1-(n-m)] x-m \alpha_{k}(t),} & x \in\left[m \alpha_{k}(t), n \alpha_{k}(t)\right], \\ (n-m)\left[\alpha_{k}(t)-x\right], & x \in\left[n \alpha_{k}(t), \alpha_{k}(t)\right] .\end{cases}
$$

Write

$$
M \triangleq \max _{(x, t) \in \widehat{Q}_{T}^{k}}|p(x, t)| .
$$


In the following, we prove (2.4) by the multiplier $p$.

Multiplying the first equation of (2.3) by $p z_{x}$ and integrating on $\left(0, \alpha_{k}(t)\right) \times(0, T)$, we obtain

$$
0=\int_{0}^{T} \int_{0}^{\alpha_{k}(t)}\left\{z_{t t} p z_{x}-z_{x x} p z_{x}\right\} d x d t \triangleq J_{1}+J_{2}
$$

We calculate the above two integrals $J_{i}(i=1,2)$. By $p(0, t)=p\left(\alpha_{k}(t), t\right)=0$ on $(0, T)$, we find the expression

$$
\begin{aligned}
J_{1}= & \int_{0}^{T} \int_{0}^{\alpha_{k}(t)} z_{t t} p z_{x} d x d t \\
= & \int_{0}^{T} \int_{0}^{\alpha_{k}(t)}\left\{\frac{\partial}{\partial t}\left[p z_{x} z_{t}\right]-p_{t} z_{x} z_{t}-p z_{x t} z_{t}\right\} d x d t \\
= & \left.\int_{0}^{\alpha_{k}(t)}\left[p z_{x} z_{t}\right] d x\right|_{0} ^{T}-\int_{0}^{T} \int_{0}^{\alpha_{k}(t)} p_{t} z_{x} z_{t} d x d t \\
& +\int_{0}^{T} \int_{0}^{\alpha_{k}(t)} p_{x} \frac{1}{2} z_{t}^{2} d x d t .
\end{aligned}
$$

Further, we have

$$
\begin{aligned}
J_{2} & =-\int_{0}^{T} \int_{0}^{\alpha_{k}(t)} z_{x x} p z_{x} d x d t \\
& =-\int_{0}^{T} \int_{0}^{\alpha_{k}(t)}\left\{p \frac{\partial}{\partial x}\left[\frac{1}{2} z_{x}^{2}\right]\right\} d x d t \\
& =-\left.\int_{0}^{T} p \frac{1}{2} z_{x}^{2}\right|_{0} ^{\alpha_{k}(t)} d t+\int_{0}^{T} \int_{0}^{\alpha_{k}(t)} \frac{1}{2} p_{x} z_{x}^{2} d x d t \\
& =\int_{0}^{T} \int_{0}^{\alpha_{k}(t)} \frac{1}{2} p_{x} z_{x}^{2} d x d t .
\end{aligned}
$$

Therefore, by (3.3) and (3.4), it follows that

$$
\int_{0}^{T} \int_{0}^{\alpha_{k}(t)} \frac{1}{2} p_{x}\left(z_{t}^{2}+z_{x}^{2}\right) d x d t=\int_{0}^{T} \int_{0}^{\alpha_{k}(t)} p_{t} z_{x} z_{t} d x d t-\left.\left[\int_{0}^{\alpha_{k}(t)} p z_{x} z_{t} d x\right]\right|_{0} ^{T}
$$

By the definition of $p$, we deduce

$$
\begin{aligned}
& \frac{[1-(n-m)]}{2} \int_{0}^{T} \int_{m \alpha_{k}(t)}^{n \alpha_{k}(t)}\left[\left|z_{t}(x, t)\right|^{2}+\left|z_{x}(x, t)\right|^{2}\right] d x d t \\
& \quad-\frac{n-m}{2} \int_{0}^{T} \int_{\left(0, \alpha_{k}(t)\right) \backslash\left(m \alpha_{k}(t), n \alpha_{k}(t)\right)}\left[\left|z_{t}(x, t)\right|^{2}+\left|z_{x}(x, t)\right|^{2}\right] d x d t \\
& =\int_{0}^{T} \int_{0}^{\alpha_{k}(t)} p_{t} z_{x} z_{t} d x d t-\left.\left[\int_{0}^{\alpha_{k}(t)} p z_{x} z_{t} d x\right]\right|_{0} ^{T},
\end{aligned}
$$

from which we obtain that

$$
\frac{1}{2} \int_{0}^{T} \int_{m \alpha_{k}(t)}^{n \alpha_{k}(t)}\left[\left|z_{t}(x, t)\right|^{2}+\left|z_{x}(x, t)\right|^{2}\right] d x d t
$$




$$
\begin{aligned}
= & \frac{n-m}{2} \int_{0}^{T} \int_{0}^{\alpha_{k}(t)}\left[\left|z_{t}(x, t)\right|^{2}+\left|z_{x}(x, t)\right|^{2}\right] d x d t \\
& +\int_{0}^{T} \int_{0}^{\alpha_{k}(t)} p_{t} z_{x} z_{t} d x d t-\left.\left[\int_{0}^{\alpha_{k}(t)} p z_{x} z_{t} d x\right]\right|_{0} ^{T} .
\end{aligned}
$$

Next, we estimate every term in the right-hand side of (3.6).

By (3.1) and (3.2), we have that

$$
\begin{aligned}
& \frac{n-m}{2} \int_{0}^{T} \int_{0}^{\alpha_{k}(t)}\left[\left|z_{t}(x, t)\right|^{2}+\left|z_{x}(x, t)\right|^{2}\right] d x d t \\
& \quad=(n-m) \int_{0}^{T} E(t) d t \\
& \left|\int_{0}^{T} \int_{0}^{\alpha_{k}(t)} p_{t} z_{x} z_{t} d x d t\right| \\
& \leq \max \{m k,(n-m) k\} \int_{0}^{T} E(t) d t \\
& \leq(n-m) k \int_{0}^{T} E(t) d t .
\end{aligned}
$$

Note that, for any $t$,

$$
\begin{aligned}
& \left|\int_{0}^{\alpha_{k}(t)} p z_{x} z_{t} d x\right| \\
& \quad \leq M E(t) \\
& \quad \leq M \frac{(1+k) \alpha_{k}(T)}{(1-k) \alpha_{k}(t)} E_{T} \\
& \quad \leq \frac{M(1+k) \alpha_{k}(T)}{1-k} E_{T} .
\end{aligned}
$$

It follows that

$$
\left|\int_{0}^{\alpha_{k}(t)}\left[p z_{x} z_{t} d x\right]\right|_{0}^{T} \mid \leq \frac{2 M(1+k) \alpha_{k}(T)}{1-k} E_{T}
$$

By (3.1), (3.6), (3.7), (3.8), and (3.9), we derive that

$$
\begin{aligned}
& \frac{1}{2} \int_{0}^{T} \int_{m \alpha_{k}(t)}^{n \alpha_{k}(t)}\left[\left|z_{t}(x, t)\right|^{2}+\left|z_{x}(x, t)\right|^{2}\right] d x d t \\
& \quad \geq(n-m)(1-k) \int_{0}^{T} E(t) d t-\frac{2 M(1+k) \alpha_{k}(T)}{1-k} E_{T} \\
& \quad \geq\left[\frac{(n-m)(1-k)^{2}}{k(1+k)} \ln (1+k T)-\frac{2 M(1+k)}{1-k}\right] \alpha_{k}(T) E_{T} .
\end{aligned}
$$

If $T>T_{k}^{*}=\frac{e^{\frac{2 k M(1+k)^{2}}{(n-m)(1-k)^{3}}}-1}{k}$, it holds that $\left[\frac{(n-m)(1-k)^{2}}{k(1+k)} \ln (1+k T)-\frac{2 M(1+k)}{1-k}\right] \alpha_{k}(T)>0$. Also,

$$
\frac{1}{2} \int_{0}^{T} \int_{m}^{n}\left[\alpha_{k}(t)\left|z_{t}(x, t)\right|^{2}+\beta_{k}(x, t)\left|z_{x}(x, t)\right|^{2}\right]
$$




$$
\begin{aligned}
& \geq\left[\frac{(n-m)(1-k)^{2}}{k(1+k)} \ln (1+k T)-\frac{2 M(1+k)}{1-k}\right] \alpha_{k}(T) E_{T} \\
& \geq C\left[\frac{(n-m)(1-k)^{2}}{k(1+k)} \ln (1+k T)-\frac{2 M(1+k)}{1-k}\right] \alpha_{k}(T)\left(\left|z^{0}\right|_{H_{0}^{1}(0,1)}^{2}+\left|z^{1}\right|_{L^{2}(0,1)}^{2}\right) .
\end{aligned}
$$

Therefore (2.4) is proved.

Remark 3.2 It is easy to check that

$$
T^{0} \triangleq \lim _{k \rightarrow 0} T_{k}^{*}=2 \max \{m, 1-n\}
$$

It is well known that (1.2) in the cylindrical domain is internally controllable at any time $T>T^{0}$. However, $T_{k}^{*}$ is not sharp.

\section{Acknowledgements}

The author wants to thank the editors and reviewers sincerely.

\section{Funding}

The author is supported financially by the National Natural Science Foundation of China (11371084) and the School Project of Jilin University of Finance and Economics (08181934).

\section{Availability of data and materials}

Not applicable.

\section{Competing interests}

The author declares that they have no competing interests.

\section{Authors' contributions}

The author read and approved the final manuscript.

\section{Authors' information}

The author comes from Jilin University of Finance and Economics of China.

\section{Publisher's Note}

Springer Nature remains neutral with regard to jurisdictional claims in published maps and institutional affiliations.

Received: 13 October 2018 Accepted: 8 April 2019 Published online: 17 April 2019

\section{References}

1. Lasiecka, I., Lions, J.L., Triggiani, R.: Non homogeneous boundary value problems for second order hyperbolic operators. J. Math. Pures Appl. 65, 149-192 (1986)

2. Lions, J.L.: Exact controllability, stabilizability and perturbation for distributed systems. SIAM Rev. 30, 1-68 (1988)

3. Zuazua, E.: Exact controllability for semilinear wave equation in one space dimension. Ann. Inst. Henri Poincaré, Anal. Non Linéaire 10, 109-129 (1993)

4. Yao, P.: On the observability inequalities for exact controllability of wave equations with variable coefficients. SIAM J. Control Optim. 37, 1568-1599 (1999)

5. Zhang, X.: Explicit observability estimate for the wave equation with potential and its application. Proc. R. Soc. Lond. A 456, 1101-1115 (2000)

6. Fu, X., Yong, J., Zhang, X.: Exact controllability for multidimensional semilinear hyperbolic equations. SIAM J. Control Optim. 46, 1578-1614 (2007)

7. Ho, L.F.: Exact controllability for the one-dimension wave equation with locally distributed control. SIAM J. Control Optim. 28, 733-748 (1990)

8. Milla Miranda, M.: Exact controllability for the wave equation in domains with variable boundary. Rev. Mat. Univ. Complut. Madr. 9, 435-457 (1996)

9. Cui, L., Liu, X., Gao, H.: Exact controllability for a one-dimensional wave equation in non-cylindrical domains. J. Math. Anal. Appl. 402, 612-625 (2013)

10. Cui, L., Song, L.: Exact controllability for a wave equation with fixed boundary control. Bound. Value Probl. 2014,47 (2014)

11. Cui, L., Gao, H.: Exact controllability for a wave equation with mixed boundary conditions in a non-cylindrical domain Electron. J. Differ. Equ. 101, 1 (2014)

12. Cui, L., Song, L.: Controllability for a wave equation with moving boundary. J. Appl. Math. 2014, 827698 (2014)

13. Sun, $H_{\text {., Li, }}$., Lu, L.: Exact controllability for a string equation in domains with moving boundary in one dimension. Electron. J. Differ. Equ. 2015, 98 (2015) 
14. Cui, L., Jiang, Y., Wang, Y.: Exact controllability for a one-dimensional wave equation with the fixed endpoint control. Bound. Value Probl. 2015, 208 (2015)

15. Bardos, C., Chen, G.: Control and stabilization for the wave equation, part III: domain with moving boundary. SIAM J. Control Optim. 19, 123-138 (1981)

16. Cui, L.: The wave equation with internal control in non-cylindrical domains. Adv. Differ. Equ. 2017, 267 (2017)

Submit your manuscript to a SpringerOpen ${ }^{\mathcal{O}}$ journal and benefit from:

- Convenient online submission

- Rigorous peer review

- Open access: articles freely available online

- High visibility within the field

- Retaining the copyright to your article

Submit your next manuscript at $\gg$ springeropen.com 